\title{
Wiederholungsfiguren in der Übersetzung Figures of repetition in translation
}

\author{
Anna Pieczyńska-Sulik \\ INSTYTUT LINGWISTYKI STOSOWANEJ, UNIWERSYTET IM. ADAMA MICKIEWICZA \\ AL. NIEPODLEGŁOŚCI 4, 61-874 POZNAŃ \\ suldamu.edu.pl
}

\begin{abstract}
This article aims to look at the problems of translating of the figures of repetition present in texts. Based on Lotman's distinction between the primary and secondary modeling system the value of repetitions in text will depend on the assignment to either one of the systems. Only in the second case will the repetitions not be blamed for redundancy, but recognized as legitimate artistic means. Whether on the phonological, morphological, syntactic or lexical level, the semiotic perspective turns out to be useful, both in terms of repetitions as well as the translation process.
\end{abstract}

\begin{abstract}
Artykuł ma na celu przyjrzenie się problemom przekładu figur powtórzenia obecnych $\mathrm{w}$ tekstach. W oparciu o łotmanowskie rozróżnienie między pierwotnym i sekundarnym systemem modelującym wartość powtórzeń $\mathrm{w}$ tekście zależeć będzie od przyporządkowania do jednego $\mathrm{z}$ systemów. Tylko $\mathrm{w}$ drugim przypadku powtórzenia nie zostaną obciążone winą redundancji, lecz mają szansę zyskać uznanie jako pełnoprawne środki artystyczne. Czy to na płaszczyźnie fonologicznej, morfologicznej, składniowej czy leksykalnej przydatnym okazuje się perspektywa semiotyczna, zarówno w odniesieniu do samych powtórzeń, jak również procesu przekładu.
\end{abstract}

\section{o.o Einleitung}

Jeder verbale Text bietet ein hohes Wiederholungspotential. Wiederholt, d.h. in unmittelbarer Nachbarschaft redupliziert bzw. in mittelbarer Nachbarschaft wiederaufgenommen werden Zeichen(teile) des primären modellbildenden Systems (Zeichen der natürlichen Sprache), wodurch sie - falls intentional wiederholt bzw. so empfangen - zu Zeichen des sekundären modellbildenden Systems (Zeichen über dem Niveau der natürlichen Sprache) erhoben werden (vgl. Lotman 1972/41993: 22). Die meistens äquivalente, seltener deviante Relation (vgl. Plett 2cooo) zwischen dem Quellen- und Wiederholungszeichen wird dabei semantisiert und evoziert neue Bedeutungen. 


\section{o.1 Wiederholungstypen}

Wie bei phonologischen Wiederholungen, d.h. Wiederholungen auf der untersten Strukturebene des Textes. Da Phoneme an sich keinen Zeichenstatus genießen, werden Lautwiederholungen entweder als sog. „leere Wörter” oder (häufiger) als semantische Relationen zwischen Wörtern herstellende Klangorganisatoren in den Rang eines Zeichens erhoben (vgl. Lotman 1972/41993: 162ff.) (z.B. Alliterationen). Morphem- und Wortwiederholungen sind dagegen Wiederholungen auf der Ebene der bedeutungstragenden Einheiten. Es handelt sich um grammatische Morphemwiederholungen (z.B. Wiederholungen von Wortwurzeln, Affixen, Flexiven) und lexikalische Morphem- und Wortwiederholungen, die ebenso verschiedene Form annehmen können (z.B. iteratio, d.h. mehrfache Nennung des gleichen Wortes). Da es eben das Wort ist, das die Grundeinheit eines Wortkunstwerks darstellt, gelten Wortwiederholungen als besonders wichtig (vgl. Lotman 1972/41993: 242). Syntagmatische Wiederholungen sind Wiederholungen von Phrasen/Syntagmen (Rekurrenz) oder syntaktischen Strukturmustern (Parallelismen) (vgl. Beaugrande/Dressler 1981: 61).

\subsection{Wiederholungswert}

Wiederholungen sind im Text unvermeidlich, weil „jeder Text als kombinatorische Verknüpfung einer begrenzten Zahl von Elementen gebildet wird“ (Lotman 1972/41993: 159). Nicht alle Wiederholungen haben jedoch denselben Wert.

\subsubsection{Aus Sicht des primären modellbildenden Systems}

Aus Sicht des allgemeinsprachlichen Mitteilungsgehalts (des primären modellbildenden Systems) sind Wiederholungen als reine Redundanz (vgl. Lotman 1972/41993: 161), aus Sicht der normativen Schulstilistik als bloße Stilfehler (vgl. Sowinski 1972/1991: 57f. ) mit dem Stigma der Zufälligkeit behaftet (vgl. auch Beaugrande/Dressler 1981:58).

\subsubsection{Aus Sicht des sekundären modellbildenden Systems}

Sobald aber die Grenze der natürlichen Sprache überschritten wird und ein Text als künstlerisch und damit als sekundäres modellbildendes System gilt mit all seinen semantisierten Geordnetheiten (vgl. Lotman 1972/41993), werden Wiederholungen zu Stilmitteln (vgl. Sowinski 1972/1991: 58) und damit „zu einem der entscheidenden Charakteristika der Textstruktur“ (Lotman 1972/41993: 161).

Wiederholungen heben dann hervor, verstärken die Intensität und lassen Wörter eindringlicher und nachdrücklicher und damit einprägsamer wirken (vgl. Sowinski 1972/1991: 60; Beaugrande/Dressler 1981: 58). Sie ziehen sich oft wie ein roter Faden durch das Werk und sind konstitutiv für die kohäsionsstiftende Isotopieebene (vgl. Beaugrande/Dressler 1981: 58; Albrecht 1998: 67). Nicht zu übersehen ist ebenso ihre komische Funktion, die von Philosophen betont wird (vgl. Bergson 1900/21991, Deleuze 1968/2007; vgl. auch Sieradzka 2007). Aus semiotischer Sicht muss das ikonische Potential von Wiederholungen (vgl. Beaugrande/Dressler 1981: 59) hervorgehoben werden, wobei hier zwischen der exophorischen 


\section{Anna Pieczyńska-Sulik: Wiederholungsfiguren in der Übersetzung Figures of repetition in translation}

(situationsbezogenen) und endophorischen (intratextuellen) Ikonizität differenziert wird (vgl. Nöth 1990: 195f.). Im Falle von exophorisch-ikonischen Wiederholungen, die Außersprachliches (Quantität, Intensität, Sequenz) (vgl. Tabakowska 1993, 1995) intra- bzw. intermedial repräsentieren (vgl. Nöth 1990: 19ff.), handelt es sich um diagrammatische Ikonizität im Sinne von Peirce, d.h. um Ähnlichkeit in Bezug auf Relationen (vgl. Nöth 2000). Im Falle der endophorischen Ikonizität, für die Wiederholungen als Conditio sine qua non gelten, handelt es sich um die bildhafte Ikonizität von Peirce, der nach gewisse Qualitäten des Wiederholten bereits im AT wiederaufgenommen werden. Endophorische Ikonizität umfasst jede Form der phonetischen (oder graphemischen) Rekurrenz, von der Phrasen-, Wort-, Morphem-, Morphemteilwiederholung (Reduplikation, Reim) bis zu einzelnen Phonemen (Alliteration). Jedes rekurrente Sprachzeichen referiert auf das vorstehende Zeichen und ist damit sein Ikon, das es wiederholt und dessen Modifikation es ist." (Nöth 1990: 195; übers. von A.P.-S.)

\section{Wiederholungen im übersetzungswissenschaftlichen Diskurs}

Auf Grund ihrer Funktion sollten Wiederholungen auch im übersetzungswissenschaftlichen Diskurs thematisiert und konsequenterweise in der Übersetzungspraxis berücksichtigt werden. Wiederholungen „haben von jeher die Aufmerksamkeit der Forscher auf sich gezogen“ (Lotman 1972/41993: 287), übersetzungswissenschaftliche Beiträge zur Wiederholungsproblematik treten jedoch eher verstreut und unsystematisiert auf. Es ist beispielsweise die komische Funktion von Wiederholungen, die wertvolle Beiträge dazu in der wortspielorientierten Literatur finden lässt (vgl. Tęcza 1994; Delebastita 1999/2006). Bezüglich der Wiedergabe von Wiederholungen werden eher, wie von Albrecht (1998: 66f.), „schwerwiegende, fast unüberwindliche Schwierigkeiten“ befürchtet, deren Ursache vorwiegend in "sprachlichen Zwängen“ liegt (vgl. auch z.B. Nida 1964). Da Zeichenträger mit Zeichenbedeutungen einerseits unzertrennlich und andererseits kontextgebunden funktionieren (d.h. je nach Kontext kann die Bedeutung variieren), sind die Voraussetzungen für funktionsgemäße Wiederholungsakte im ZT tatsächlich beschränkt gegeben. Auch wenn (typologische) Unterschiede zwischen der Ausgangsund Zielsprache nicht allzu gravierend sind, leisten verschiedene Wiederholungsfälle translatorischen Widerstand. Was die rein semantischen Wiederholungen betrifft, scheint die Sache relativ einfach $\mathrm{zu}$ sein, da jede Sprache über Paraphrasierungsmöglichkeiten verfügt, die zwar nie identische, aber doch nicht allzu entfernte Bedeutungen tragen können. Nicht viel schlimmer verhält es sich mit syntaktischen Wiederholungen. Am schlimmsten sieht es mit partiellen ATWiederholungen aus, bei denen der semiotische Träger des Quellenzeichens wiederholt, von dessen Bedeutung jedoch beim Wiederholungszeichen abgewichen wird. Das wortspielerische Verfahren ist unabhängig vom Umfang des zu wiederholenden Zeichens und reicht von der Morphem-, Wort-, Phrasen, Satz- bis zur Textwiederholung. Was die Phonemebene anbetrifft, wo die Bedeutung erst durch die Rekurrenz eines Phonems auf ein andres aktualisiert wird, ist die Sache nicht viel leichter. Im Allgemeinen ist die Wahrscheinlichkeit, auch bei sog. vollständigen Wiederholungen, dass in der Zielsprache Zeichen(folgen) (Phoneme, Morpheme, Wörter, Phrasen, Sätze, Texte) vorhanden sind bzw. kombiniert werden können, mit denen der jeweilige AT-Wiederholungsakt analog sinnstiftend im ZT rekonstruiert werden kann, relativ gering. Auf das sog. heureka-Verfahren 
angewiesen, dem die Peirce'sche Abduktion zugrunde liegt (vgl. Gorlée 2004), müssen also Übersetzer permanent auf der Kompensierungssuche sein. In Frage kommt hier entweder die Versetzung der durch Rekurrenz semantisierten Stellen (vgl. die versetzte Äquivalenz bei Rei $\beta$ 1971), oder die Abweichung von der ATReferenz der markierten Sprachformen. „Auch ganz subtile Eigenschaften des sprachlichen Materials können [nämlich] wichtiger sein als die referentielle Bedeutung, z.B. bei Alliterationen, lautmalerischen Effekten usw.“ (Lipiński 1989: 215).

Es kommt aber auch vor, dass der Übersetzer die Wiedergabelösungen von der ZS sozusagen geschenkt bekommt und es kommt eben darauf an, die nicht zu übersehen. Neben sprachlichen Zwängen ist nämlich auch oft die „Unachtsamkeit“ (Albrecht 1998: 67) des Übersetzers die Ursache der stiefmütterlichen Behandlung von Wiederholungen. Das ist auch der Punkt, wo die Übersetzungsdidaktik das meiste zu tun hat. Da die AT-Rezipienten von künstlerischen Texten - der Reorganisation der Abbildungsstrategie auf dem Oberflächentext zufolge - mehr Aufmerksamkeit auf Rekurrenzen konzentrieren (vgl. Beaugrande/Dressler 1981: 59), sollten auch die ZTRezipienten die Möglichkeit erhalten, die AT-Invariante (vgl. Lipiński 1989; Barańczak 1994) erkennen und im ZT genießen zu können. Helfen kann dabei ein semiotischer Gesichtspunkt.

Wenn man beispielsweise die Art der semiotischen Organisation des Quellen- und des Wiederholungszeichens (vgl. Szczęsna 2007: 58) überlegt, kommt man zu der Überzeugung, dass Texte polysemiotisch sind. Die natürliche Sprache ist zwar ein monosemiotisches System, ein in der natürlichen Sprache formulierter Text dagegen nicht mehr. Ein semiotisch nuancierter Blick erkennt die zumindest zweifache Kodierung (gesprochen vs. schriftlich) und damit die Polysemiotizität eines Textes. „Die Sprache, zumindest die ursprüngliche, die gesprochene Sprache, ist, in ihrem Ausdruck betrachtet, gestalteter Schall; (...) Die geschriebene Sprache, wiederum in ihrem Ausdruck betrachtet, ist demgegenüber Gestalt, ,Zeichnung'“ ' Coseriu 1994: 111f.) Der Schall unterliegt dem Kriterium der Linearität, die Zeichnung wird simultan auf der zweidimensionalen Textfläche rezipiert. Geschriebene Sprachzeichen nehmen verschiedene Silhouetten an, wodurch ihre Sprachkörper zusätzlich zu Bildkörpern werden. Dadurch wird die Ebene der Grapheme ästhetisch, manchmal auch - wenn auch seltener - kommunikativ relevant (vgl. Lipiński 1989: 214f.). Der schweizerische Typograph, Adrian Frutiger (2006: 25), behauptet, „dass alle Zeichen in Form eines Buchstabens schwerer als Bilder zu registrieren sind, da im Unterbewussten des Betrachters diese Figuren schon als Buchstaben vorhanden sind und eine andere Vorstellung praktisch ausschließen." Dies mag ja für eine nicht sprachliche Umgebung zutreffen. In einem Text jedoch, d.h. in einer sprachlichen Umgebung (Kontext) fallen andere Eigentümlichkeiten auf als in einer nicht sprachlichen Umgebung, darunter die Rekurrenz gewisser Zeichenträger (Silhouetten eben), die zueinander - durch gleiche oder ähnliche Linienführung - in einer visuell wahrnehmbaren endophorisch-ikonischen Relation stehen. In einer auditiv wahrnehmbaren Relation stehen (dieselben) Zeichen, wenn sie gesprochenen werden. Damit sind textuelle Wiederholungen - wie die Texte selbst - polysemiotisch und dies sollte - nach Möglichkeit - im Übersetzungsprozess berücksichtigt werden. $\mathrm{Zu}$ der Beibehaltung der endophorischen Ikonizität von Zeichensilhouetten ebenso im ZT sollte die diagrammtisch-ikonische Wiedergabe von AT-Wiederholungen 


\section{Anna Pieczyńska-Sulik: Wiederholungsfiguren in der Übersetzung Figures of repetition in translation}

verhelfen, was ohne entsprechendes Bewusstsein der Übersetzer aus Unachtsamkeit vernachlässigt werden kann.

\section{Wiedergabepotential von Wiederholungen aus semiotischer Sicht}

Im Folgenden sollte ein Versuch unternommen werden, Wiederholungen aus semiotischer Sicht auf ihr Wiedergabepotenzial hin zu untersuchen, und zwar mit der Überzeugung, dass der semiotische Ansatz die erwähnten Unachtsamkeiten zumindest zum Teil reduzieren lässt. Die Semiotisierung des Wiederholungsphänomens ist mit dem semiotischen Ansatz in der Übersetzungswissenschaft kompatibel, dem nach Texte als komplexe Zeichen und der Übersetzungsprozess als Semioseakt gilt (vgl. z.B. Petrilli 2003; Gorlée 1994, 2004). Dank der Verankerung in der Semiotik von Ch. S. Peirce gelten Zeichen der natürlichen Sprache als eine Zeichenart unter vielen, wodurch ihnen dieselbe allgemein semiotische Charakteristik zukommt wie auch allen übrigen Zeichen. Als (komplexe) Zeichen sind also Texte im Sinne von Peirce triadisch zu bestimmen, was (unter anderem) heißt, dass sie in Bezug auf ihr Objekt (hier: Ausgangstext) indexikalisch, ikonisch und symbolisch sind (vgl. Gorlée 1994: 170ff.; Ponzio 2003: 15; Petrilli 2003a: 24f., 2007: 324ff.; vgl. auch Pieczyńska-Sulik 2009). In Bezug auf den AT sollten Wiederholungen diagrammatisch-ikonisch wiedergegeben werden, innerhalb des ZT - dem AT analog - endophorisch-ikonisch bleiben.

Die Erfahrung mit übersetzten Texten zeigt jedoch, dass Wiederholungen im Übersetzungsprozess oft stiefmütterlich behandelt werden, und zwar nicht unbedingt nur in Fällen, wo die sprachlichen Zwänge wirksam sind. Als Beispiel sollten hier vier Ausschnitte aus einer der Kindergeschichten von Peter Bichsel, Ein Tisch ist ein Tisch, mit ihren polnischen Pendants aus der polnischen Fassung von Krystyna Müller, Stół to stól, übersetzungskritisch konfrontiert werden.

Die Textwahl geht auf die formale Schlichtheit und Nüchternheit (vgl. Bucheli 200o: 82f.) der Bichselschen Kindergeschichten zurück, für die Wiederholungen auf allen Sprachebenen konstitutiv sind. Bekanntlich ist das Kurzprosastück eine philosophisch untermauerte Babel-Geschichte eines alten Mannes, der Haushaltsgegenstände umbenennt, womit er eine arbiträre und konventionelle Ordnung durch eine andere, ebenso arbiträre, aber nicht mehr konventionelle ersetzt und der konsequenterweise außerstande ist, sich mit anderen zu verständigen.

\section{(1) (T:26)}

Dem Bett sagte er Bild.

Dem Tisch sagte er Teppich.

Dem Stuhl sagte er Wecker.

Der Zeitung sagte er Bett.

Dem Spiegel sagte er Stuhl.

Dem Wecker sagte er Fotoalbum.

Dem Schrank sagte er Zeitung.

Dem Teppich sagte er Schrank.

Dem Bild sagte er Tisch. (1a) $(S: 27)$

A więc łóżko będzie „obrazem“.

Stół to „dywan”.

Krzesło to „budzik”.

Gazeta to „łóżko”.

Lustro to „krzesło”.

Budzik to „album do zdjęć”.

Szafa to "gazeta”.

Dywan to „szafa”.

Obraz to „stól”. 
Und dem Fotoalbum sagte er Spiegel.

\section{(2) (T:28f.)}

Seinem Bild sagen die Leute Bett.

Seinem Teppich sagen die Leute Tisch.

Seinem Wecker sagen die Leute Stuhl.

Seinem Bett sagen die Leute Zeitung.

Seinem Stuhl sagen die Leute Spiegel.

Seinem Fotoalbum sagen die Leute Wecker.

Seiner Zeitung sagen die Leute Schrank.

Seinem Schrank sagen die Leute Teppich.

Seinem Tisch sagen die Leute Bild.

Seinem Spiegel sagen die Leute Fotoalbum.

\section{(3) (T:26)}

Am Morgen blieb der Mann lange im Bild liegen, um neun läutete das Fotoalbum, der Mann stand auf und stellte sich auf den Schrank, damit er
A album do zdjęć będzie odtąd „lustrem”.

(1b) (A.P-.S.)

Odtąd lóżko to obraz./lawa

Odtąd stół to dywan./sofa

Odtąd krzesło to budzik.

Odtąd gazeta to łóżko./Odtąd prasa to łóżko.

Odtąd lustro to krzesło.

Odtąd budzik to album.

Odtąd szafa to gazeta./Odtąd szafa to prasa.

Odtąd dywan to szafa.

Odtąd obraz to stól.

I odtąd album to lustro.

(2a) (S:30)

Na jego obraz ludzie mówią „łóżko”.

Na dywan ludzie mówią „stół”.

Na budzik ludzie mówią „krzesło”.

Na łóżko ludzie mówią „gazeta”.

Na krzesło ludzie mówią „lustro”.

Na album do zdjęć ludzie mówią „dywan”.

Na gazetę ludzie mówią „szafa”.

Na szafę ludzie mówią „dywan”.

Na stół ludzie mówią „obraz”.

Na lustro ludzie mówią „album do zdjęć”.

(2b) (A.P-.S.)

Jego obraz to dla ludzi łóżko.

Jego dywan to dla ludzi stół.

Jego budzik to dla ludzi krzesło.

Jego łóżko to dla ludzi gazeta/prasa/książka.

Jego krzesło to dla ludzi lustro.

Jego album to dla ludzi dywan.

Jego gazeta to dla ludzi szafa.

Jego szafa to dla ludzi dywan.

Jego stół to dla ludzi obraz.

Jego lustro to dla ludzi album.

(3a) $(\mathrm{S}: 27)$

Stary Człowiek długo leżał w obrazie, o dziewiątej zadzwonił album do zdjęć, Człowiek wstał, stanął na szafie, żeby mu nie zmarzły nogi, potem wyjął 


\section{Anna Pieczyńska-Sulik: Wiederholungsfiguren in der Übersetzung Figures of repetition in translation}

nicht an die Füße fror, dann nahm er seine Kleider aus der Zeitung, zog sich an, schaute in den Stuhl an der Wand, setzte sich dann auf den Wecker an den Teppich und blätterte den Spiegel durch, bis er den Tisch seiner Mutter fand. ubrania z gazety, ubrał się, spojrzał w krzesło na ścianie, potem usiadł na budziku przy dywanie i przeglądał lustro, dopóki nie znalazł stołu swej matki.

\section{(4) (T:27)}

läuten heißt stellen

frieren hei $\beta$ t schauen

liegen hei $\beta$ t läuten

stehen heißt frieren

stellen hei $\beta$ t blättern.

\author{
(4a) (S:28) \\ Dzwonić to „stawiać” \\ Marznąć to „patrzeć” \\ Leżeć to „dzwonić” \\ Stać to „marznąć” \\ Stawać to „przeglądać”.
}

(4b) (A.P-.S.)

dzwonić znaczy stawiać

marznąć znaczy patrzeć

leżeć znaczy dzwonić

stać znaczy marznąć

stawiać znaczy przeglądać

Auf die beiden Ordnungen wird im AT mit Parallelismen exophorisch-ikonisch verwiesen, darunter mit einem rhetorisch-anaphorischen Beginn aufeinander folgender Sätze ((1), (2)). In (1) und (2) stehen alle Sätze wie mit dem Lineal gezogen, in (1a) dagegen ist die Ordnung zumindest dreifach gestört: durch die fehlende Anaphorisierung, durch die zweifache Abweichung von dem sonst stabilen Strukturmuster mit der Prädikativparikel to „X to $\mathrm{Y}^{\text {“ }}$ und - was dessen Konsequenz ist - durch den Einsatz von gebeugten Nomina (obrazem, lustrem) an der Stelle, wo Jonglieren mit unveränderten Benennungsetiketten diagrammatisch-ikonisch nachgeahmt werden sollte. So was tut man nicht einem Text, dessen Autor von sich gesagt hat, er sei zum Schreiber durch das Lesen von B u c h s t a b e n und W ö r t e r n geworden (vgl. Schlosser/Zimmermann 1998: 232; Hervorh. von A.P.-S.). Es ist ziemlich paradox, dass die vom AT (1) gegebenen visuellen Signale (gleiche Wortund ähnliche Satzsihouetten) ignoriert werden, während der ZT ((1a), (2a), (3a)) unnötigerweise mit Anführungszeichen optisch belastet wird, ein diesmal - wie es scheint - wohl überlegtes Verfahren zur Kennzeichnung des Metasprachlichen, womit aber die Übersetzerin päpstlicher sein will als der Papst.

In (2a) wurde zwar die Visualität des AT (2) zumindest zum Teil erkannt, damit aber wird der Parallelismus der ersten Aufzählung (1) nicht aufgegriffen, wodurch wiederum der AT-Spiegelbildeffekt zerstört wird (Dem Bett sagte er Bild./ Seinem Bild sagen die Leute Bett.) Außerdem schützt die eingesetzte präpositionale Anapher die auftretenden femininen Nomina (gazetę, szafę) nicht vor Beugung, worunter wiederum der ikonische Effekt der Umstellung von Wortfiguren leidet. Der ikonische Effekt leidet ebenso unter der Auslassung - wahrscheinlich aus schulstilistischen Gründen - des im AT rekurierrenden Possessivums. Zwar haben Artikelwörter, darunter Possessiv-Artikel, im Deutschen und im Polnischen einen anderen Wert, 
hier dient jedoch der wiederholte Einsatz des Possessivums einem übergeordneten Zweck. Und der Zweck heiligt die Mittel, oder sollte die zumindest heilen.

In (1b) und (2b) wird ein Versuch unternommen, die erwähnten Unzulänglichkeiten des bestehenden Zieltextes zu beseitigen, und zwar durch den jeweiligen Einsatz ausnahmsloser Parallelismen, den anaphorischen Beginn aufeinander folgender Sätze sowie typographische Reinhaltung des Textes. Natürlich haben die ATAnaphern (der bestimmte und der Possessiv-Artikel) einen anderen Wert als das ziemlich penetrant wirkende Adverb odtąd oder das Possessivum jego. Da aber Wiederholung die absolute Invariante des Textes darstellt, scheinen die vorgeschlagenen Lösungen - der semiotischen Treue gemä $\beta$ - gerechtfertigt zu sein. Optional könnte noch in (1b) ein auf Alliteration und Wortlänge (gleiche Silbenzahl) bedachter Lexemersatz auf Kosten der Referenz (z.B. Bett - Bild vs. łóżko - lawa) überlegt werden. Da hier aber die Alliteration kein durchgehend eingehaltenes Prinzip darstellt, muss sie nicht als sinnstiftend eingeschätzt werden. Und die gleiche Silbenzahl (z.B. lóżko (zweisilbig) vs. prasa/książka (zweisilbig) statt gazeta (dreisilbig)) würde als ein zusätzliches rhythmisierendes ZT-Verfahren dienen, das die anderen Verluste ausgleicht. Da in (2b) die Prädikativpartikel to eingesetzt wurde, wurde für die in (4) enthaltenen Parallelismen eine andere Lösung vorgeschlagen (4b) als in (4a).

Der Forschungsehrlichkeit wegen sollte dabei zugegeben werden, dass die Aufrechterhaltung der ungebeugten Wortformen (3) die ganze Geschichte durch unmöglich ist, und zwar wegen des Flexionszwangs polnischer Nomina (3a). Im Folge der Umbenennung werden nämlich die neuen Wortetiketten in konkreten Sätzen gebraucht, deren primäre konventionelle Bedeutung durch die angedeuteten syntaktischen Strukturen erschließbar ist. In einer Sprache mit der stark entwickelten Flexion, wie es das Polnische ist, ist hier der Verzicht auf die Flexion nicht möglich, aber auch - wie es scheint - weniger nötig, weil hier nicht der Umbenennungsakt selbst signiert wird, sondern lediglich der Gebrauch der neuen Zeichen.

\section{Fazit}

Sinnstiftende Wiederholungen gehören im literarischen Text zum sekundären modellbildenden System. Im Falle der Wiederholung des Zeichenträgers handelt es sich um „das basale Faktum der schriftlichen Visualität, der Präsenz von Graphemen auf einer Fläche“ (Witte 2003: 375), über die im Übersetzungsprozess nicht hinweggesehen werden darf. Besonders schade ist es, wenn die unnötige Zerbröckelung der Sprachmaterie nicht auf „sprachliche Zwänge“ zurückgeht, sondern auf die „Unachtsamkeit“ des Übersetzers. Im Übersetzungsprozess geht nämlich zu viel verloren (vgl. Barańczak 1994), als dass man sich noch Unachtsamkeit leisten könnte. 


\section{Anna Pieczyńska-Sulik: Wiederholungsfiguren in der Übersetzung Figures of repetition in translation}

\section{Literatur}

Barańczak, St. 1994. Ocalone w tłumaczeniu. Szkice o warsztacie tłumacza w poezji z dołączeniem małej ontologii przekładów. Poznań: Wydawnictwo a5.

Beaugrande, R.-A., Dressler, W. U. 1981. Einführung in die Textlinguistik. Tübingen: Max Niemeyer Verlag.

Bergson, H. L. 1900, 21991. Das Lachen. Ein Essay über die Bedeutung des Komischen. Aus dem Französischen von R. Plancherel-Walther. Frankfurt am Main: Luchterhand.

Bichsel, P. 1969,1997. Ein Tisch ist ein Tisch. in: Bichsel, P. Kindergeschichten. Frankfurt am Main: Suhrkamp Verlag, pp. 21-30, (= T).

Bichsel, P.2007. Stółtostół. in: Bichsel, P.2007.Dziecinnehistorie. übers. von K. Müller. Kraków: Wydawnictwo SOLURA, pp. 21-31, (= S).

Bucheli, R. 2000. Die melancholische Poesie der Hauptsätze. Gedanken zur Bodenlosigkeit in Peter Bichsels Prosa. In: Hoven, Herbert (Hg.): In Olten umsteigen. Über Peter Bichsel. Frankfurt am Main: Suhrkamp Verlag, pp. 80-89.

Coseriu, E. 1994. Textlinguistik. Eine Einführung. [Hrsg. und bearb. von Jörn Albrecht]. Tübingen/Basel: Francke Verlag.

Delebastita Dirk (1999,22006): Wortspiele. Aus dem Englischen von Paul Kußmaul. in:

Snell-Hornby, Mary et al. (Hg.): Handbuch Translation. Tübingen: Stauffenburg Verlag, 285288.

Deleuze, G. 1968, 2007. Differenz und Wiederholung. Aus dem Französischen von J. Vogl. München: Wilhelm Fink Verlag.

Frutiger, A. 2006. Der Mensch und seine Zeichen. Wiesbaden: Marix Verlag.

Gorlée, D. L. 1994.Semiotics and the Problem of Translation. With Special Reference to the Semiotics of Charles S. Peirce. Amsterdam/Atlanta: Radopi.

Gorlée, D. L. 2004. On Translating Signs. Exploring Text and Semio-Translation. Amsterdam, Atlanta: Radopi.

Lipiński, K. 1989.Über die Sonderstellung der literarischen Übersetzung. in: Kątny, A. (Hg.): Studien zur kontrastiven Linguistik und literarischen Übersetzung. Frankfurt am Main: Peter Lang Verlag, pp. 211-220.

Lotman, J. M. 1972,41993. Die Struktur literarischer Texte. München: Wilhelm Fink Verlag.

Nida, E. A. 1964. Toward a Science of Translating. With Special Reference to Principles and Procedures Involved in Bible Translating. Leiden: E.J. Brill.

Nöth, W. 1990. The semiotic potential for iconicity in spoken and written language. in: Kodikas/Code 13, pp. 191-209.

Nöth, W. 2000. Handbuch der Semiotik. Stuttgart,Weimar: Verlag J.B. Metzler.

Petrilli, S. Hg. 2003. Translation translation. Amsterdam/New York: Rodopi.

Petrilli, S. 2003a. Translation and Semiosis. Introduction. in: Petrilli, S. (Hg.), pp. 17-36.

Petrilli, S. 2007. Interpretive trajectories in translation semiotics. in: Semiotica $163-1 / 4$, pp. 311-345.

Pieczyńska-Sulik, A. 2009. O semiotyce przekładu. in: Rocznik Przekładoznawczy 5, pp. 153163.

Plett, H. F. 2000. Systematische Rhetorik. München: Wilhelm Fink Verlag.

Ponzio, A. 2003.Preface. Aus dem Italienischen von S. Petrilli. in: Petrilli, Susan (Hg.) (2003), 13-16.

Schlosser, H. D., Zimmermann, H. D. (Hg.). 1988. Poetik. Frankfurt am Main: Athenäum Verlag.

Sieradzka, Małgorzata (2007): Wiederholung als sprachlich-stilistisches Mittel im Besuch der alten Dame und in der polnischen Fassung der tragischen Komödie Friedrich Dürrenmatts. In: StudiaGermanicaResoviensia 5, 11-35.

Sowinski, Bernhard (1972/1991): Deutsche Stilistik. Beobachtungen zur Sprachverwendung und Sprachgestaltung im Deutschen. Frankfurt am Main: Fischer Taschenbuchverlag. 
Szczęsna, Ewa (2007): Poetykamediów. Polisemiotyczność, digitalizacja, reklama. Warszawa Wydział Polonistyki Uniwersytetu Warszawskiego.

Szczęsna, Ewa (2007a): Powtórzenie i polisemiotyczność. Typy i formy powtórzeń. In: Przegląd Humanistyczny 2 (2007), 57-71.

Tabakowska, Elżbieta (1993): Cognitive Linguistics and Poetics of Translation. Tübingen: Gunter NarrVerlag.

Tabakowska, Elżbieta (1995): Gramatyka i obrazowanie. Wprowadzenie do językoznawstwa kognitywnego. Kraków: PAN.

Tęcza, Zygmunt (1994): Das Wortspiel in der Übersetzung. Stanisław Lems Spiele mit dem Wort als Gegenstand interlingualen Transfers. Tübingen: Max Niemeyer Verlag.

Witte, Georg (2003): Textflächen und Flächentexte. Das Schriftsehen der literarischen Avantgarden. In: Grube, Gernot et al. (Hg.): Schrift. Kulturtechnik zwischen Auge, Hand und Maschine. München: Wilhelm Fink Verlag, 375-396. 